\title{
Laboreal
}

Volume $6 \mathrm{~N}^{\circ} 2$ | 2010

Varia

\section{Seveso : el desastre y la Directiva}

Seveso : o desastre e a Directiva

Seveso : le désastre et la Directive

Seveso: the disaster and the Directive

\section{Laura Centemeri}

\section{(2) OpenEdition}

\section{Journals}

Edición electrónica

URL: https://journals.openedition.org/laboreal/8950

DOI: $10.4000 /$ laboreal.8950

ISSN: 1646-5237

\section{Editor}

Universidade do Porto

Referencia electrónica

Laura Centemeri, «Seveso : el desastre y la Directiva», Laboreal [En línea], Volume 6 No2 2 2010,

Publicado el 01 octubre 2010, consultado el 21 septiembre 2021. URL: http://journals.openedition.org/ laboreal/8950 ; DOI: https://doi.org/10.4000/laboreal.8950

Este documento fue generado automáticamente el 21 septiembre 2021.

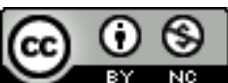

Laboreal está licenciado com uma Licença Creative Commons - Atribuição-NãoComercial 4.0 Internacional. 


\title{
Seveso : el desastre y la Directiva
}

\author{
Seveso : o desastre e a Directiva \\ Seveso : le désastre et la Directive \\ Seveso : the disaster and the Directive
}

Laura Centemeri

1 Séveso es un pequeño Municipio italiano, situado cerca de $22 \mathrm{~km}$ al norte de Milán, ciudad capital de la región de Lombardía. Séveso es parte del denominado "Distrito del Mueble" de Brianza, un territorio con tradición católica que se refleja en su cultura y su política [ $\left.{ }^{1}\right]$.

2 El nombre Séveso está asociado, en Italia y en Europa, al accidente industrial que aconteció el 10 de Julio de 1976, causando una contaminación con Dioxina en gran parte de su territorio y a los Municipios limítrofes de Cesano Maderno, Desio y Meda. Estamos haciendo alusión a la mayor exposición de TCDD [2], registrada hasta el momento a una población humana (Eskenazi y col., 2001). Todavía hoy se efectúan estudios epidemiológicos para analizar las consecuencias sanitarias del accidente. Séveso le dio nombre a la Directiva Europea sobre Riesgos de Accidentes Graves (Directiva 82/501/ CEE del 24 de Junio de 1982).

\section{El desastre}

3 El accidente que dio origen al desastre de Séveso ocurrió en la fábrica de químicos ICEMSA, situada en Meda. La fábrica era propiedad de la multinacional farmacéutica suiza Roche. El sábado 10 de Julio de 1976, alrededor de las $12: 30 \mathrm{pm}$, el reactor donde se producía el triclorofenol, un componente intermedio utilizado en la preparación de herbicidas y de una sustancia antibacteriana (el hexaclorofeno), liberó una nube tóxica de dioxina y otros contaminantes como consecuencia de una reacción exotérmica inesperada. La nube depositó estos venenos sobre el territorio compuesto por Meda, Cesano Maderno, Desio y Séveso. Debido a la dirección del viento, el territorio de Séveso fue el más alcanzado. 
4 De acuerdo a la Comisión Parlamentar que investigó el desastre de Séveso, el accidente estuvo directamente relacionado con la falta de inversiones adecuadas para la seguridad de las instalaciones de la fábrica [3]. Roche estaba al tanto de los riesgos de la producción de triclorofenol que ya habían acontecido en casos anteriores de accidentes industriales. Riesgos que se derivaban de la dioxina, sustancia que se produce como residuo durante la transformación de triclorofenol.

5 En 1976 los estudios epidemiológicos sobre los efectos de la dioxina eran pocos y se limitaban a casos de obreros (hombres y adultos) expuestos accidentalmente a elevadas concentraciones de dioxina (Zedda, 1976), y evidenciaban, a pesar de esto, la existencia de efectos extremadamente nocivos de esta sustancia sobre la salud humana. La contaminación de todo un territorio y su población era un evento sin precedentes. Además, no existía cómo medir la presencia de dioxinas en el cuerpo humano (Mocarelli, 2001). De esta forma, estaban frente a una condición de "incertidumbre radical" (Callon Barthe \& Lascoumes, 2001) sobre la extensión y posibles consecuencias de la contaminación. Estas consecuencias, sin embargo, ya se concebían como catastróficas.

6 La catástrofe no se reconoció de inmediato. Los habitantes de Séveso y de Meda estaban acostumbrados a los olores molestos emitidos a veces por la que irónicamente había sido bautizada como "la fábrica de los perfumes". Entonces ocurrió una "semana de silencio" (Fratter, 2006), Roche por fin anunció el 19 de Julio que el accidente de ICMESA había causado una fuga de dioxina y sugirió la evacuación de la población del área más gravemente afectada. Los responsables de dar respuesta a la crisis fueron las autoridades de la Región de Lombardía, con el apoyo de las autoridades estatales.

7 La evacuación comenzó el 24 de Julio, 736 habitantes de Séveso y de Meda fueron obligados a dejar sus casas, sin la posibilidad de llevar consigo cualquier objeto personal. De ellos, 200 no pudieron regresar a sus casas, que fueron destruidas durante los trabajos de limpieza. Paralelamente, una Comisión de Especialistas dividió el territorio afectado en "zonas de riesgo" basado en la trayectoria estimada de la nube, pero, sobre todo, teniendo como referencia los criterios de factibilidad y sustentabilidad social de una evacuación de gran tamaño [4]. El resultado fue una cartografía de la contaminación en la que las zonas de riesgo eral delimitadas por líneas rectas, coincidiendo con los límites administrativos o naturales, lo que hizo que los ciudadanos tuviesen dudas sobre la lógica que estaba por detrás del uso de estos criterios.

8 Sin embargo, el tema que suscitó más conflicto fue la decisión de las autoridades regionales de autorizar abortos terapéuticos a las mujeres embarazadas (que se encontraban en el límite del tercer mes de gestación) residentes en la zona contaminada; teniendo de base a los posibles efectos teratógenos de la dioxina. En aquel tiempo, el debate sobre la despenalización del aborto estaba en el centro de la escena política italiana. El caso de Séveso catalizó la atención de los principales actores del conflicto, lo que hizo que fuese todavía más trágico y sufrido el dilema para algunas mujeres de Séveso, en un ambiente que, recordemos, tenía una fuerte componente católica (Ferrara, 1977) [5].

9 La forma en que la Región de Lombardía respondió a la crisis estuvo marcada por un elevado grado de centralización en la decisión. Se crearon Comisiones de Especialistas encargadas de proporcionar las soluciones necesarias fundadas en decisiones unánimes. El Consejo Regional pretendía con esto poder limitarse a ratificar la decisión 
técnica. De este modo, cuestiones de naturaleza inextricablemente política, científica y social, se redujeron a problemas técnicos (Conti, 1977).

La centralización de la decisión, así como su bajo nivel de democracia, explican los conflictos que surgieron al momento de la implementación de las medidas tomadas, vistos como resistencias por parte de la población. La necesidad de una mayor participación de la población afectada por la decisión, se reivindicó tanto por los movimientos sociales nacionales, movilizados en Séveso para denunciar los costos del capitalismo usando un discurso de crítica social ; como por las Comisiones espontáneas de ciudadanos, creadas, en la mayoría de los casos, alrededor de las parroquias del territorio. Los Movimientos Nacionales y las Comisiones espontáneas se dividían, sin embargo, en el tema de los abortos terapéuticos, cuestión que acabó monopolizando el espacio público (Centemeri, 2006).

Donde los Movimientos y las Comisiones se unieron, fue en la respuesta a la decisión de la Región de Lombardía de construir en la Zona A un incinerador donde serían quemados los restos derivados de los trabajos de descontaminación. Los ciudadanos, en cambio, presentaron un proyecto para enterrar los residuos en dos vertederos sanitarios especiales a ser construidos en la Zona A, pues su deseo era ver a la Zona A transformada en un parque urbano. Después de dos años de conflicto, la Región de Lombardía decidió apoyar el proyecto de los ciudadanos.

Entre 1981 y 1984 se cavaron dos vertederos en la Zona A y la descontaminación se terminó en Diciembre de 1985. En 1984 comenzaron los trabajos para reforestar la Zona A, transformándola en un parque urbano - el "Bosque de los Robles" - que fue abierto al público en 1996. En el 2004 se inauguró la "Ruta de la Memoria en el Bosque de los Robles", con once paneles que cuentan la historia del desastre y el origen del bosque. Se trata del resultado de un trabajo complejo de escritura colectiva por parte de la comunidad afectada, para la memoria del desastre [ $\left.{ }^{6}\right]$.

13 Se puede decir que, desde el punto de vista del diálogo entre ciudadanos e instituciones, Séveso no fue un buen ejemplo debido a la alta tasa de conflictos que caracterizó la crisis y la respuesta a la crisis. Sin embargo, la recuperación ambiental del territorio fue un éxito. Paralelamente a la recuperación ambiental, la zona afectada recuperó rápidamente las condiciones socio-económicas que caracterizaban al territorio antes de la contaminación.

14 Hoy, Séveso es una ciudad donde varias organizaciones y asociaciones se empeñan en actividades de recuperación y valorización ambiental, aunque el desastre continúa presente en la realidad local con el tema las consecuencias sanitarias se mantiene abierto.

15 La producción científica sobre los efectos de la dioxina en Séveso resultó significativa. Sin embargo, esta producción no fue objeto de discusión entre la población afectada, cuyo grado de participación en los estudios estuvo limitado a responder cuestionarios o a someterse a idas periódicas al médico (Centemeri, forthcoming). Además, estos estudios acabaron por marginalizar a los objetivos de salud pública del territorio pues se concentraron, a partir de Séveso, en la exploración de mecanismos biomoleculares que explicaban los peligros de la dioxina en el ser humano.

16 Los estudios epidemiológicos confirmaron la variedad de los efectos de la dioxina sobre la salud de la población afectada. Variedad que se consideró en términos de grados de gravedad y de frecuencia (Consonni y col., 2008; Baccarelli y col., 2008), apoyando de 
esta forma a la clasificación que se hizo en 1997 por parte de la International Agency for Research on Cancer (IARC) que reconoció a la dioxina como siendo cancerígena (grupo 1) para el ser humano (Steeland, Bertazzi, Maccarelli \& Kogevinas, 2004).

\section{La(s) Directiva(s) "Séveso" : gestión de riesgo y governance}

17 El accidente en Séveso fue un evento determinante para la definición de una regulación a nivel europeo sobre riesgos de esta naturaleza, y que acabó por incluirse en la Directiva relacionada con los riesgos de accidentes graves en ciertas actividades industriales (82/501/CEE), conocida como "Séveso 1".

¿Cómo se explica entonces que la Comunidad Económica Europea, exactamente a seguir el accidente de Séveso, hubiese afirmado la urgencia de una regulación europea sobre riesgos de actividades industriales? Hubo para ello varias razones. Una de las razones más fuertes fue seguramente la naturaleza transnacional del accidente ocurrido en ICMESA, pero también lo fue el hecho de que el accidente ocurriese en Italia, al sur de Europa. Era de conocimiento común en los "medios comunitarios" de Bruselas que la regulación italiana, sobre todo en materia de ambiente y seguridad industrial, estaba atrasada con relación a los países del norte de Europa (van Eijndhoven, 1994).

Sin embargo, el Tratado CEE, en su versión original de 1957, no previa la existencia de poderes y de competencias que permitiesen a la Comisión el tener una iniciativa legislativa en materia de riesgos industriales en el ámbito de una política de tutela ambiental [7]. El camino seguido fue a través de la armonización de las Legislaciones y Reglamentos de los Estados-Miembro sobre este tema, fundamentado por el hecho de que la divergencia existente estaba "provocando una distorsión en las condiciones de competición del mercado común" (Artículo 100 del Tratado). Además, fue a través de esta base legal, que permitió cubrir la carencia de poderes de la CEE en varios dominios, que se adoptaron a partir de 1973 las primeras Directivas concernientes a la defensa del ambiente.

20 La Directiva 'Séveso 1 ' se concentró en aspectos del "risk management" que se operacionalizaron a través de obligaciones relacionadas con la información. Aquí es donde se ve reflejada la influencia directa del acontecimiento en Séveso : el accidente de ICMESA se considera, de hecho, un "desastre de información" (van Eijndhoven, 1994). La cuestión que se destacó, a partir de la experiencia del accidente, fue la de la falta de información necesaria para que la población afectada y las autoridades responsables pudiesen actuar oportunamente después del accidente. De hecho, la Directiva pretendió promover una armonización de los reglamentos nacionales, haciendo de la comunicación un elemento de seguridad fundamental relacionado con este tema.

21 En este sentido, la parte más innovadora de la Directiva es la que consta en su Artículo $8^{\circ}$, relacionada con la información al público, y que constituye una obligación que puede debilitar el secreto industrial relacionadas con las actividades incluidas. Simultáneamente, la Directiva diseñó una especie de red de información entre las autoridades públicas y las industrias, y entre las industrias y las partes que se encuentran en riesgo potencial (Otway, 1990 ; Otway y Amendola, 1989). 

en la aplicación de las Legislaciones en vigor, se adoptó en 1996 una nueva Directiva (Directiva 96/82/CEE del 9 de Diciembre de 1996) que substituyó a la "Séveso 1" y que fue reconocida como la Directiva "Séveso 2". Entre las modificaciones más importantes que se incluyeron en esta nueva Directiva, aparecieron nuevas demandas que comenzaron a incidir en la planificación del territorio como un elemento integrante en la prevención de accidentes graves, actuando coherentemente con la preocupación por focalizarla en la protección del ambiente.

El verdadero cambio en la Directiva "Séveso 1" estuvo en el modo como se consideró a la información y a la comunicación pública. En muchos de los Artículos de la Directiva "Séveso 2", particularmente en su Artículo 13ํㅗㅇ se consagró el reconocimiento de darle a la población un papel activo en su "derecho a la participación", aunque todavía en una etapa embrionaria. del Parlamento Europeo y del Consejo, del 16 de Diciembre del 2003 ("Séveso 3"). El motivo de estos cambios estuvo en el accidente que se produjo el 21 de Septiembre del 2001 en la fábrica de químicos AZF en Tolosa, una instalación clasificada como "Séveso". Esta Directiva pasó a incluir las operaciones de procesamiento y almacenaje de las materias minerales producidas por las industrias extractoras, relacionadas con sustancias peligrosas; y cuya innovación fundamental fue la de prolongar el cumplimiento de las obligaciones de la gestión a las empresas sub-contratadas que trabajasen en los establecimientos incluidos en la normativa "Séveso".

Con las Directivas "Séveso 2" y "3", el concepto de gestión de riesgos de esta naturaleza se transformó, de un problema técnico reservado, en principio, a los especialistas, configurándose e instituyéndose progresivamente en un problema de governance (De Marchi, Pellizzoni \& Ungaro, 2001). Y, en el caso de la Unión Europea, se relacionó con cuestiones de 'Gobierno' como la "libre circulación de trabajadores y la política social, las condiciones de trabajo, la seguridad de los trabajadores, el ambiente, los consumidores y la protección a la salud, la contaminación y las perturbaciones, las sustancias químicas, los riesgos industriales y la biotecnología".

\section{BIBLIOGRAFÍA}

Baccarelli, A., Giacomini, S.M., Corbetta, C., Landi, M.T., Bonzini, M., Consonni, D., Grillo, P., Patterson, D.G., Pesatori, A.C. \& Bertazzi, P.A. (2008). Neonatal Thyroid Function in Seveso 25 Years after Maternal Exposure to Dioxin, PLoS Medicine, 5 (7): e161, 1133-1142.

Bagnasco, A. (1977). Tre Italie : la problematica territoriale dello sviluppo italiano. Bologna : Il Mulino.

Callon, M., Barthe, Y. \& Lascoumes, P. (2001). Agir dans un monde incertain. Essai sur la démocratie technique, Paris : Seuil.

Centemeri, L. (2006). Ritorno a Seveso. Il danno ambientale, il suo riconoscimento, la sua riparazione, Milano : Bruno Mondadori.

Laboreal, Volume $6 \mathrm{~N}^{\circ} 2$ | 2010 
Centemeri, L. (2010). The Seveso disaster's legacy, in M. Armiero, M. Hall (dir.), Nature and History in Modern Italy. Athens (OH): Ohio University Press \& Swallow Press, pp. 251-273.

Centemeri, L. (forthcoming). What kind of knowledge is needed about toxicant-related health issues? Some lessons drawn from the Seveso dioxin case, in S. Boudia e N. Jas, Powerless Science? The Making of the Toxic World in the Twentieth Century, Oxford and New York: Berghahn Books.

Consonni, D., Pesatori, A.C., Zocchetti, C., Sindaco, R., Cavalieri D’Oro, L., Rubagotti, M. \& Bertazzi, P.A. (2008). Mortality in a Population Exposed to Dioxin after the Seveso, Italy, Accident in 1976: 25 Years of Follow-Up, American Journal of Epidemiology, 167, 847-858.

Conti, L. (1977). Visto da Seveso : l'evento straordinario e l'ordinaria amministrazione, Milano : Feltrinelli.

De Marchi, B., Pellizzoni, L. \& Ungaro, D. (2001). Il rischio ambientale, Bologna : Il Mulino.

Diamanti, I. (2009). Mappe dell'Italia politica, Bologna : Il Mulino.

Eijndhoven, J. van (1994). "Disaster prevention in Europe." in S. Jasanoff (dir.), Learning from disaster. Risk Management After Bophal, Philadelphia: University of Pennsylvania Press, 113-132.

Eskenazi, B., Mocarelli, P., Warner, M., Samuels, S., Needham, L., Patterson, D., Brambilla, P., Gethoux, P.M., Turner, W., Casalini, S., Cazzaniga, M. \& Chee, W.Y. (2001). Seveso Women's Health Study: Does Zone of Residence Predict Individual TCDD Exposure? Chemosphere, 43, 937-942.

Ferrara, M. (1977). Le donne di Seveso, Roma : Editori Riuniti.

Fratter, M. (2006). Memorie da sotto il bosco, Milano : Auditorium.

Otway, H. (1990). Communicating with the public about major hazards: challenges for European research, in H.B.F. Gow, H. Otway (a cura di), Communicating with the public, New York: Elsevier.

Otway, H. \& Amendola, A. (1989). Major hazard information policy in the European Community: Implications for risk analysis, Risk Analysis, 9, 4, 505-512.

Steenland, K., Bertazzi, P. A. Maccarelli, A. \& Kogevinas, M. (2004). Dioxin Revisited: Developments Since the 1997 IARC Classification of Dioxin as a Human Carcinogen, Environmental Health Perspectives, 112(13), 1265-1268.

Mocarelli P. (2001). Seveso: A Teaching Story, Chemosphere, 43, 391-402.

Sambeth, J. (2004). Zwischenfall in Seveso, Zurich : Unionsverlag

Zedda,S. (1976). La lezione della cloracne, in AA.VV. Icmesa. Una rapina di salute, di lavoro e di territorio, Milano : Mazzotta.

\section{NOTAS}

1. Sobre el "Distrito Industrial", ver el trabajo fundador de Bagnasco (1977). Sobre el mapa político de Italia y su desarrollo relacionado con la división que se mantuvo hasta inicios de los años noventa en regiones "rojas" (donde gobernaba el Partido Comunista) y regiones "blancas" (donde gobernaba la Democracia Cristiana), ver en Diamanti (2009).

2. La 2,3,7,8-tetraclorodibenzo-p-dioxina (TCDD), conocida como Dioxina de Séveso, es un subproducto de varias reacciones químicas y procesos de combustión a base de cloro orgánico.

3. Informe concluyente de la Comisión Parlamentar de investigación sobre la fuga de sustancias tóxicas ocurrido el 10 de Julio de 1976 en las instalaciones de ICMESA y sobre los riesgos potenciales para la salud y el ambiente, que se derivan de actividades industriales: Actos 
Parlamentares, VII legislautra, doc. XXIII, n.6, 1978; véase también el libro autobiográfico del Exdirector Técnico Givaudan Jörg Sambeth (2004).

4. La Zona A (108 hectáreas) fue evacuada. La Zona B (269 hectáreas, 4600 habitantes) no fue evacuada debido a que las concentraciones de dioxina registradas fueron consideradas como compatibles con la posibilidad de continuar habitando el territorio. Los habitantes fueron obligados a respetar estrictas normas de conducta. La Zona de Respeto (1 430 hectáreas, 31800 habitantes) no fue evacuada, porque las concentraciones de dioxina eran limitadas. Sin embargo, sus habitantes tuvieron que seguir algunas normas de precaución.

5. La interrupción voluntaria del embarazo fue despenalizada con la Ley 194 de 1978.

6. Acerca de la redacción de los paneles del recorrido de la memoria, ver en Centemeri (2006, 2010). Los paneles puede consultarse en el sitio web: http://www.boscodellequerce.it/ pubblicazioni/Informazioni/Informazioni.asp?ID_M=178

7. Es con el Acto Único de 1986, que modifica el Tratado CEE, donde se fijan los fundamentos jurídicos para la intervención de la Comunidad Europea en materia ambiental (Artículos 130r, 130s y 130t).

\section{ÍNDICE}

Temas: o Dicionário

\section{AUTOR \\ LAURA CENTEMERI}

Centro de Estudos Sociais, Universidade de Coimbra, Colégio de S. Jerónimo, Apartado 3087, 3001-401 Coimbra, Portugal

centemeri@ces.uc.pt 